Nazareth Gallego Morón

Universidad Pablo de Olavide, Sevilla, España

\title{
Discriminación de Género en el Sistema Universitario Argentino
}

\begin{abstract}
Resumen: Las mujeres son mayoría entre el alumnado y el cuerpo docente de las universidades argentinas. No obstante, estas aparecen agrupadas principalmente en ciertas áreas y apenas acceden a las categorías superiores. Se constata una discriminación horizontal y vertical. Nuestro objetivo consiste en estudiar la participación de las mujeres y profundizar en la percepción acerca de la discriminación de género. En primer lugar, se realiza un análisis descriptivo del cuerpo docente de la Universidad Nacional de Salta (UNSa). En segundo lugar, se analizan los resultados de una encuesta aplicada a la plantilla femenina de la Facultad de Ingeniería de la UNSa. Se confirma una baja percepción y visibilización del problema, especialmente por parte de las profesoras de menor edad, siendo señaladas ciertas barreras como los principales obstáculos en su carrera profesional.
\end{abstract}

Palabras Clave: Mujeres, Segregación ocupacional, Ciencia, Argentina.

\section{Introducción}

Poco más de un siglo ha pasado desde que, en 1885, Élida Passo se convirtiera en la primera mujer argentina egresada universitaria (Alicia I. PALERMO, 2006). A pesar del paso del tiempo y del avance que se ha producido en el reconocimiento de derechos, la realidad y los datos estadísticos muestran que aún queda un largo camino por recorrer hacia la igualdad.

Numerosos/as autores/as e informes institucionales han mostrado cómo en la mayor parte de los países del mundo, aunque la participación de las mujeres en la educación superior universitaria es mayor que la de los hombres, estas acceden en menor proporción a la cúspide de la pirámide organizacional y se agrupan en aquellas áreas y campos considerados tradicionalmente femeninos (Janeen BAXTER y Erik Olin WRIGHT, 2000; Consuelo FLECHA y Patricia I. PALERMO, 2008; Vicente LLORENT y Verónica COBANO, 2017; Marta PANAIA, 2014; Loreto REBOLLEDO y Ma Paulina ESPINOZA, 2016).

De este modo, la segregación ocupacional femenina se produce complementariamente en dos niveles: horizontal y vertical. Por un lado, podemos diferenciar la segregación

\section{(c) (i)}

Esta obra está sob licença Creative Commons. 
horizontal, que se define como la situación en la que existe una clara "separación de los mercados de trabajo masculino y femenino de manera que hombres y mujeres se encuentran en distintas ocupaciones y tipos de actividad" (Olga SALIDO, 2002, p. 10). Las mujeres se agrupan en aquellas que suponen una extensión del trabajo doméstico y de cuidados.

En la actualidad, se continúa diferenciando ocupaciones y estudios considerados tradicionalmente femeninos y aquellos otros masculinos, aptos para un sexo u otro, basándose en arraigados roles y estereotipos de género que operan desde las primeras etapas de la socialización ( $M^{a}$ Elina ESTÉBANEZ, 2010). Discriminaciones de género que se producen en el mercado de trabajo argentino y se repiten en la universidad, traduciéndose en peores condiciones laborales, niveles mayores de desempleo $-y$, por lo tanto, de inestabilidad-, menor valoración social (Ester BARBERÁ, Amparo RAMOS, Maite SARRIÓ y Carlos CANDELA, 2002) y brechas salariales de género que, como se desprenden de los datos estadísticos, no se pueden explicar como causa de un menor nivel educativo de las mujeres (Sofía ROJO y Lucía TUMINI, 2008).

Por otro lado, las estadísticas son claras y concisas, las mujeres acceden en menor medida a las categorías superiores más altas de la universidad, a pesar de constituirse como una mayoría entre los egresos. Tal y como muestra Ana GUIL (2008) en un estudio realizado en países de Europa, América y Asia, donde se analiza la presencia de las mujeres entre el alumnado y el profesorado, el fenómeno del 'techo de cristal' (glass ceiling) se caracteriza por su universalidad, independientemente de que las mujeres obtengan mejores calificaciones que los hombres.

Según los últimos datos disponibles, ofrecidos por el informe She Figures realizado por la COMISIÓN EUROPEA (2016), en la UE-28, en 2013 las mujeres representaban el 20,9\% del total del personal docente e investigador correspondiente al más alto nivel de la carrera académica (Grado A). La presencia femenina oscilaba entre el $11 \%$ en Chipre hasta el $61 \%$ de Macedonia. Los países con mayores porcentajes de mujeres, junto a Macedonia, eran Malta (45\%) y Croacia (38\%). En sintonía con la media europea se situaban países como Austria $(20,3 \%)$, España $(20,9 \%)$ e Italia $(21,1 \%)$. En el lado opuesto, junto a la ya citada Chipre, se encontraban la República Checa donde las mujeres pertenecientes a la categoría universitaria más alta únicamente constituían el 13\% y Lituania con un 14\%.

Una de las principales preocupaciones de la literatura internacional ha consistido en el diagnóstico y análisis de las causas del techo de cristal en la Academia. Para el caso de Nigeria, Christina O. OGBOGU (2010) realizó 730 encuestas a mujeres de doce universidades, identificando como principales barreras los problemas de conciliación como consecuencia de las cargas derivadas de la maternidad y el matrimonio. Por su parte, Belinda PROBERT (2005) coincide al señalar el matrimonio y la familia, junto con otras barreras culturales, como los roles de género y la sociedad machista en la universidad australiana.

Para el caso de España, la literatura concluye que los problemas de conciliación de la vida personal, laboral y social (Susana ANTÓN, 2005; Ana GUIL, 2005; Ana PUY, 2008; Marina TOMÁS y Cristina GUILLAMÓN, 2009), junto con la existencia e influencia de redes informales de poder en el sistema universitario (Concepción FERNÁNDEZ, 1989; Esther ESCOLANO, 2006; Ma Antonia GARCÍA DE LEÓN, 1990; Inés SÁNCHEZ, Sara DE LA RICA y Juan José DOLADO, 2011) se constituyen como las barreras más recurrentes entre el discurso de las profesoras e investigadoras universitarias. En esta misma línea, Dora BARRANCOS (2011) plantea la homogamia característica del sistema científico argentino.

En Estados Unidos, Lotte BAILYN (2003) mostró la existencia de desigualdades de género en un estudio realizado en el Masachussets Institute of Technology (MIT). A través del análisis de currículos, concluyó la preferencia por los candidatos hombres frente a las 
mujeres. Del mismo modo, comparó salarios, mostrando la existencia de una brecha salarial de género. En 2009, Jerlando F.L. JACKSON y Elizabeth M. O'CALLAGHAN realizaron una revisión de 66 investigaciones sobre techo de cristal en Estados Unidos, en la que concluyeron que la menor presencia de las mujeres en las categorías superiores no podía ser explicada según los méritos que estas poseían.

La bibliografía es coincidente al señalar una baja percepción del problema y concienciación por parte del profesorado universitario (María José ALONSO, 2002; Ma Antonia GARCÍA DE LEÓN y Ma Luisa GARCÍA DE CORTÁZAR, 2001; Ana GUIL, Ana SOLANO y Manuela ÁLVAREZ, 2005). Hombres y mujeres suelen negar la existencia de discriminación en la universidad. Además, esta situación es invisibilizada en mayor parte por el profesorado más joven y/o con menor antigüedad, quien percibe con menor intensidad este problema (Inma PASTOR, Ángel BELZUNEGUI, Blanca MORENO y Carmen MAÑAS, 2010; Eulalia PÉREZ, $M^{a}$ Jesús SANTESMASES y Paloma ALCALÁ, 2005).

De esta realidad se desprende la relevancia de este artículo. Tantos los datos estadísticos como la literatura confirman la existencia de discriminación de género en la Universidad. Sin embargo, en palabras de Georgeta Ion, $\mathrm{M}^{a}$ del Mar Durán y $\mathrm{M}^{a}$ Dolores Bernabeu (2013, p. 136), "existe una brecha entre lo oficialmente escrito y lo realmente vivido". A pesar de las evidencias, las propias protagonistas, las mujeres docentes e investigadoras, no son conscientes de la problemática. Siguiendo el planteamiento realizado por Mauricio Matus y Nazareth Gallego (2015, p. 616):

Para implementar políticas de concienciación o de intervención es necesario tener un mapa claro de cuáles son las percepciones del profesorado y particularmente si realmente existen estas diferencias en las percepciones por sexo, categoría o área de conocimiento. Sin esta información la efectividad de políticas de concienciación o intervención será muy baja y por supuesto ineficiente.

Partiendo de investigaciones anteriores, nuestro estudio ha sido diseñado para la investigación de las percepciones de las mujeres docentes acerca de la discriminación de género y de las barreras y obstáculos que han tenido o tienen que superar a lo largo de su carrera académica.

\section{Metodología y Objetivos}

El presente trabajo se contextualiza en la Universidad Nacional de Salta (UNSa), fundada en 1972. Esta se constituye como una de las universidades más feminizadas de Argentina, gozando de una proporción de mujeres docentes algo superior a la media nacional; $54 \%$ y $48,9 \%$, respectivamente (UNSa, 2015 y SISTEMA RHUN, 2013).

Son dos los objetivos principales. Por un lado, el primero de ellos consiste en conocer la participación de las mujeres (estudiantes y docentes) en la UNSa, en general, y en la Facultad de Ingeniería, en particular, con el fin de estimar la existencia o no de la segregación horizontal y/o vertical. Por otro lado, se pretende analizar las percepciones de las docentes acerca de la discriminación de género y de las barreras y obstáculos que han tenido o tienen que superar a lo largo de su carrera académica, tanto en el acceso como durante los procesos de promoción. Además, se considera la existencia de significancias estadísticas en las percepciones de estas según su edad.

Se aplicó una metodología cuantitativa, aunque acompañada de una revisión y análisis bibliográfico previo que permitiera conocer el estado de la cuestión en Sistema Universitario Nacional en Argentina. En primer lugar, se ofrece un análisis descriptivo de la participación de hombres y mujeres en la Universidad Nacional de Salta y en la Facultad 
de Ingeniería. Para ello, se han analizado datos estadísticos secundarios provenientes de dos fuentes de información: los datos proporcionados por el Departamento de Estadísticas Universitarias de la UNSa y los disponibles en la página web de la Secretaría de Políticas Universitarias (SPU).

Para el segundo objetivo, la herramienta de investigación utilizada fue una encuesta simple. Durante el proceso de diseño y validación se contó con la colaboración de profesorado de la Universidad Pablo de Olavide (Sevilla) y la Universidad Nacional de Salta (Argentina). Para ello, se realizó un pretest a modo de prueba piloto, tras el cual algunas preguntas fueron adaptadas y modificadas, asegurando la validez y calidad de la encuesta.

La muestra no es aleatoria. El universo lo ha conformado el total personal docente femenino de la Facultad de Ingeniería, estando conformada por 116 mujeres, lo que supone el $42,2 \%$ del total del profesorado de la Facultad de Ingeniería. Se obtuvo una tasa de respuesta del 19\%. La categoría académica con mayor tasa de respuesta corresponde a las mujeres profesoras (mayores de 40 años de edad), donde la muestra alcanzó el 38,5\% de la población. En las auxiliares docentes la participación fue del 9,1\%.

Se considera que la muestra no es representativa. A pesar de ello, la baja tasa de respuesta por parte del profesorado coincide con otros estudios realizados con anterioridad como es el caso de Antón (2005) para el total de la Universidad de Alicante, Guil, Solano y Álvarez (2005) para la Comunidad Autónoma de Andalucía, o Tomás y Guillamón (2009) para cuatro universidades catalanas. Puede ser considerado como una muestra más de la escasa concienciación y sensibilidad hacia este tema por parte de las propias docentes, tal y como apunta la literatura existente. Además, los resultados de la investigación señalan en la misma dirección que los mencionados, por lo que puede ser considerado como un antecedente para futuros estudios.

La encuesta consistió en un total de siete preguntas acerca de la percepción de la discriminación de género en la Universidad y las causas del techo de cristal. La naturaleza de las preguntas era la siguiente: tres de carácter dicotómico (sí/no), dos cerradas categorizadas, una numérica consistente en una Escala Likert donde debían valorar de 1 a 4 (correspondiendo 1 a menos y 4 a más importancia) un listado de 15 barreras y obstáculos según consideraban el grado en el que habían afectado o afectaban en su carrera académica a la hora de promocionar; y una última abierta, donde las docentes podían incluir cualquier comentario que considerasen oportuno.

Los datos fueron codificados y tratados a través del programa informático estadístico SPSS, cruzando la variable sexo con la edad y escala docente. Para ello, se diferenciaron tres grupos de edad: menores de 40 , de 40 a 50 y mayores de 50 años de edad. Cabe confirmar que los tramos de edad establecidos coinciden a su vez con la escala de las docentes: auxiliar ayudante y profesorado, siendo todas aquellas menores de 40 años auxiliares docentes, por lo que durante el análisis de los resultados únicamente aparece recogida la variable edad.

\section{La participación de las mujeres en la Universidad Nacional de Salta}

Los cambios socioeconómicos y culturales producidos a lo largo del siglo XX en Argentina influyeron en el marcado crecimiento en la participación de mujeres en la educación universitaria durante la década de mediados de los sesenta y mediados de los ochenta (PALERMO, 1998). En ese período el porcentaje de mujeres tituladas pasó de constituir el $30 \%$ al 50\%, igualando el porcentaje masculino (Graciela MAGLIE y Mónica FRINCHABOY, 
1988). A pesar de esta evolución, las mujeres seguían agrupándose en un reducido número de estudios, encontrando limitaciones tanto en el acceso a áreas tradicionalmente masculinizadas así como a cargos directivos y de decisión (Susana V. GARCíA, 2006).

Casi cuatro décadas más tarde, el porcentaje de mujeres que cursan estudios universitarios es superior al de hombres. Así, en el año 2014 el alumnado femenino matriculado en los estudios de pregrado y grado en las universidades argentinas representaba el $57,2 \%$, y el $61,5 \%$ de egresos, según datos de la Secretaria de Políticas Universitarias (SPU, 2014). No obstante, mientras que en campos como las Ciencias de la Salud o Ciencias Humanas en grado y pregrado las mujeres representaban el $73 \%$ y el $69,4 \%$ del total de matriculaciones, respectivamente; en Ciencias Aplicadas -donde se incluyen carreras como las ingenierías o informáticas- el porcentaje caía hasta el 36,3\% (SPU, 2014).

Sin embargo, esta superioridad de alumnas no se ve equiparada con el acceso femenino a la docencia universitaria, ya que en el año 2013 el cuerpo docente del Sistema Nacional Universitario Argentino estaba conformado por un 48,9\% de mujeres, frente al $51,1 \%$ de hombres. Por su lado, en la cúspide de la pirámide universitaria la cifra de hombres titulares casi duplicaban a las mujeres: el $38,8 \%$ del total de cargos titulares estaban ocupados por las mujeres y el $61,2 \%$ por hombres. En lo que respecta a su presencia en los cargos y puestos de responsabilidad en las autoridades superiores, en el año 2013 , las mujeres únicamente ocupaban el $10,4 \%$ del total de rectorados/presidencias de las Universidades Nacionales Argentinas. En términos absolutos, se traduce en la existencia únicamente de cinco mujeres rectoras en un total de 50 universidades (SISTEMA RHUN, 2013).

La escasa representación de mujeres en los altos cargos de poder y puestos de responsabilidad ha sido definido como el fenómeno del 'techo de cristal' (glass ceiling). Se trata de un concepto que surge en la década de los ochenta en EEUU, haciendo referencia a una barrera invisible, acotada y sólida (Ann M. MORRISON, Randall WHITE y Ellen VAN VELSOR, 1987, p. 13) que dificulta o impide la promoción de las mujeres a lo largo de la carrera profesional en igualdad de condiciones que sus compañeros varones. Así pues, a pesar del aumento de matrículas y egresos femeninos en la universidad argentina, actualmente continúa existiendo una clara discriminación de género, que se manifiesta, por un lado, en la discriminación horizontal del alumnado y el profesorado, junto, por otro lado, con la discriminación vertical.

Centrándonos en la Universidad Nacional de Salta (UNSa), las mujeres conformaban una mayoría en el año 2014 tanto en los estudios de pregrado y grado con un $54,4 \%$ de matrículas y $55,4 \%$ de egresos, como en las maestrías $(53,8 \%$ de las matrículas). Además, en los estudios de doctorado el porcentaje de estudiantes ascendía hasta el $66,4 \%$ del total y el $56 \%$ de lecturas de tesis doctoral (SPU, 2014).

Si analizamos el cuerpo docente de la UNSa, estaba formado, en el año 2015, por 2.260 personas, de las que 1.220 eran mujeres y 1.040 eran hombres. De este modo, las mujeres representaban el $54 \%$ del total. Estas se distribuían en un $66 \%$ en la escala de auxiliares y un $34 \%$ en la de profesorado $-62,3 \%$ y $37,7 \%$ de hombres, respectivamente(UNSa, 2015). Se observa cómo a pesar de que se constituían como una mayoría entre el cuerpo docente, estas se agrupaban en mayor medida que los hombres en los puestos bases de auxiliares.

La mayor presencia de mujeres entre los/as auxiliares docentes configura un fenómeno conocido como 'suelos pegajosos' (sticky floor) y que hace referencia a la existencia de dificultades que deben superar las mujeres graduadas incluso para acceder a la carrera académica en sus primeros niveles (Obdulia TORRES y Bernadette PAU, 2012). 
Metafóricamente, parece que las mujeres caminasen sobre un suelo que las atrae y les impide continuar avanzando, encontrándose sobre representadas en la base de la pirámide organizacional.

En la Gráfica 1 se aprecia el momento posterior a la lectura de la tesis doctoral y la transición hasta el acceso a la carrera académica, convirtiéndose ese momento en un punto clave en el futuro profesional de las mujeres. Los datos muestran el paso de una mayoría femenina entre el alumnado -incluso durante el momento de la realización de estudios de doctorado en el que el porcentaje de mujeres doblaba al de hombres $166,4 \%$ y $33,6 \%$, respectivamente)-, a una representación de menos de la mitad del total del profesorado titular (48,6\% de mujeres y $51,4 \%$ de hombres), para los años $2014 / 2015$.

Gráfica 1: Distribución de hombres y mujeres en la carrera estudiantil (2014) y académica en la Universidad Nacional de Salta (2015).

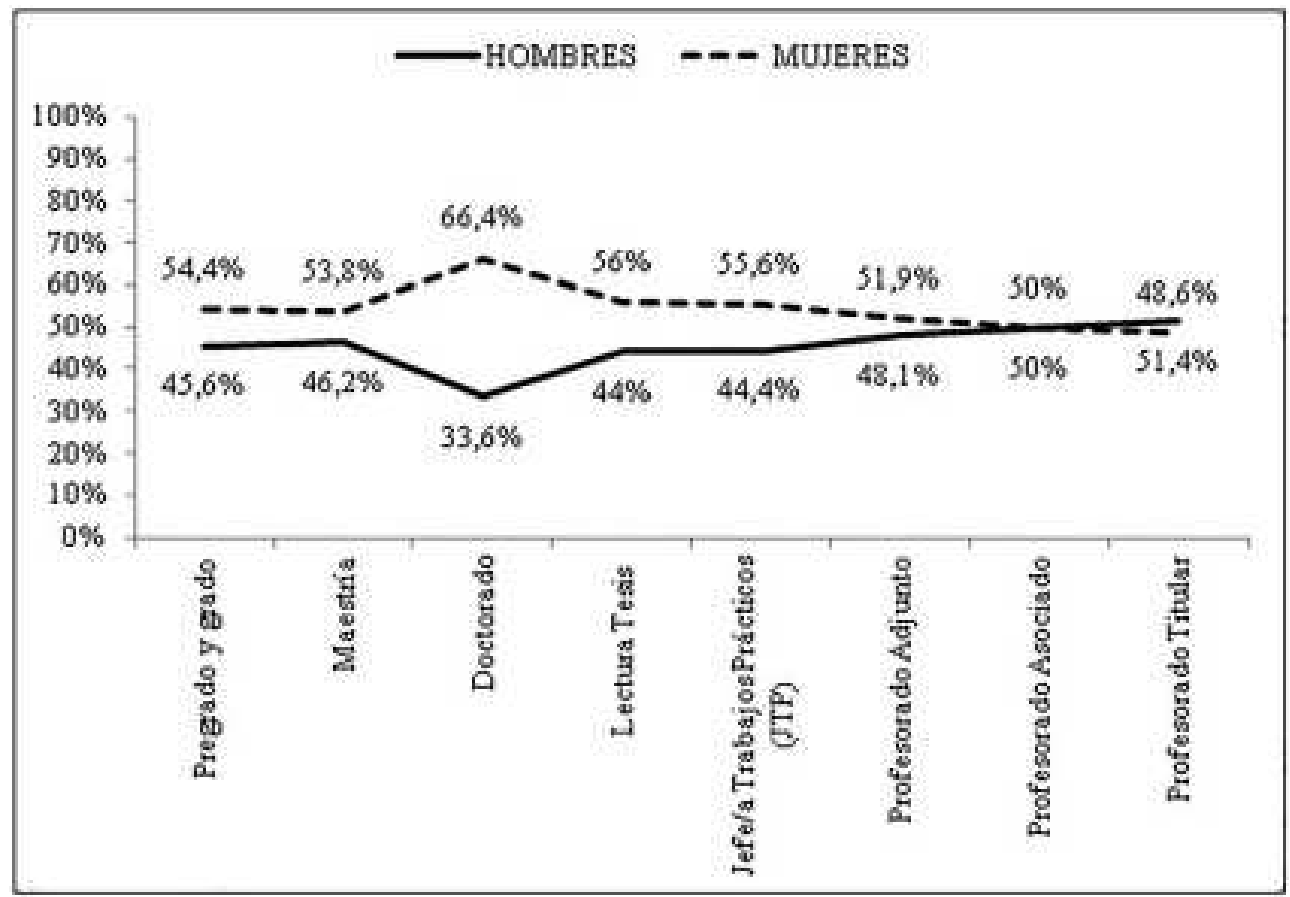

Fuente: Elaboración propia sobre datos del SPU (2014) y UNSa (2015).

De este modo, a pesar de que la cifra de mujeres que comienzan sus estudios de pregrado y grado en la UNSa es mayor que la de hombres, son progresivamente adelantadas por sus compañeros a lo largo de la carrera. Estas pasan de configurar el $54 \%$ del total de docentes universitarios/as al 51,9\% del profesorado adjunto. Si avanzamos un escalafón más, el porcentaje cae hasta el $50 \%$ del total del profesorado asociado.

Finalmente, en la cúspide de la pirámide la cifra de hombres titulares superaban a las mujeres en tres puntos. Aunque prácticamente se produce una situación de igualdad, desde el inicio de la carrera el número de hombres ha ido aumentando en detrimento de sus compañeras. Como se muestra en la Gráfica 1, la participación de estas disminuye en casi 
siete puntos porcentuales desde el cargo de jefas de trabajos prácticos $(55,6 \%)$-puesto superior en la escala de auxiliares docentes-, hasta el de titulares $-48,6 \%$ - (UNSa, 2015).

Es lo que ha venido a denominarse leaky pipeline o 'tubo que pierde', término utilizado para hacer alusión al "flujo desproporcionado de mujeres que salen del sistema profesional en el campo de la ciencia, la investigación y la docencia superior" (Flora DE PABLO, 2006, p. 2). En ocasiones también ha sido definido como la 'tesis de la pipeta', recurriendo a la misma metáfora, ya que "a medida que avanza el flujo a lo largo de la pipeta surgen barreras de diferente naturaleza que producen la exclusión de las mujeres" (María Elina ESTÉBANEZ, Daniela DE FILIPPO y Alejandra SERIAL, 2003, p. 9).

\section{La Facultad de Ingeniería de la Universidad Nacional de Salta: segregación horizontal}

Tradicionalmente, la Ciencia - en general -, y la ingeniería -en particular-, se han constituido como ámbitos caracterizados por una hegemonía masculina, donde las mujeres han estado escasamente representadas. Actualmente, esta situación se mantiene. La existencia de una socialización diferenciada, de estereotipos y roles de género junto con un cúmulo de barreras y obstáculos personales, organizacionales y sociales, hacen que la participación de las mujeres en el ámbito de la ciencia y la tecnología sea muy baja. ${ }^{1}$

Si nos detenemos a analizar la Facultad de Ingeniería, ámbito donde se desarrolla el presente estudio, observamos cómo se presentan una serie de particularidades con respecto a la situación de las mujeres en la UNSa, descrita anteriormente. En primer lugar, como hemos mencionado, de manera general las estudiantes comienzan siendo una mayoría entre el alumnado universitario en pregrado, grado y posgrado, tanto en matrículas como en egresos. Sin embargo, en el caso de la Facultad de Ingeniería, las estudiantes comienzan constituyéndose como una minoría.

En las Ciencias Aplicadas en la UNSa, las mujeres únicamente representaban en el año 2014 el 32\% del total de estudiantes y el 31,6\% del total de egresos en estudios de pregrado y grado. No obstante, el porcentaje asciende hasta el $62,5 \%$ del total de estudiantes en las maestrías y egresos de doctorado en este campo (SPU, 2014). Se constata que las alumnas que consiguen superar la segregación ocupacional existente se encuentran altamente capacitadas y cualificadas para continuar siendo una mayoría en la carrera académica e investigadora.

En segundo lugar, la discriminación horizontal se reproduce entre el cuerpo docente. Con respecto al total de docentes femeninas de la UNSa, en el año 2015, únicamente el $9,5 \%$ pertenecía a este campo, frente al 15,3\% de hombres. Sin embargo, las cifras se invierten en áreas como Humanidades, donde se situaba el 15,1\% del total de mujeres docentes, duplicando al $7,8 \%$ de varones.

Atendiendo concretamente a la plantilla docente de la Facultad de Ingeniería de la UNSa, esta estaba formada por un total de 275 docentes, de los cuales 116 eran mujeres $(42,2 \%)$ y 159 hombres $(57,8 \%)$. Del total de las 116 profesoras, 77 pertenecían al cuerpo de auxiliares docentes $(66,4 \%)$ y 39 al de profesorado $(33,6 \%)$. Únicamente seis mujeres ocupaban el puesto de profesora titular, el máximo cargo (UNSa, 2015).

De este modo, los suelos pegajosos continúan produciendo efecto, repitiéndose una mayor acumulación de mujeres en los puestos bases, es decir, entre los/as auxiliares

\footnotetext{
1 Para más información se aconseja consultar el Informe "Mujeres que hacen ciencia y tecnología en Argentina: una década de avances y retos pendientes" (Gloria BONDER; Anabella BENEDETTl; Patricia FLORES y Paula MAGARIÑOS, 2013).
} 
docentes -en comparación al cuerpo de profesorado-, que estaba conformado por un $43 \%$ de mujeres y $57 \%$ de hombres. En el caso del total del profesorado, el porcentaje de mujeres disminuía hasta el 40,6\%, siendo superado por los varones por casi 20 puntos, con un $59,4 \%$ (UNSa, 2015).

Finalmente, si analizamos la composición por sexos de las diferentes categorías académicas de la Facultad de Ingeniería (Gráfica 2) obtenemos un gráfico tipo 'tijera', al igual que sucedía en la Gráfica 1. No obstante, los porcentajes correspondientes a la participación de ambos sexos en los distintos cargos se invierten en comparación con la situación general de la plantilla total de la UNSa. Del mismo modo en que el alumnado femenino era notablemente inferior en los primeros niveles universitarios, las mujeres comienzan siendo una minoría simbólica en el acceso a la carrera: los cargos de auxiliares docentes.

Gráfica 2: Distribución de hombres y mujeres en la carrera académica de la Facultad de Ingeniería de la UNSa, 2015.

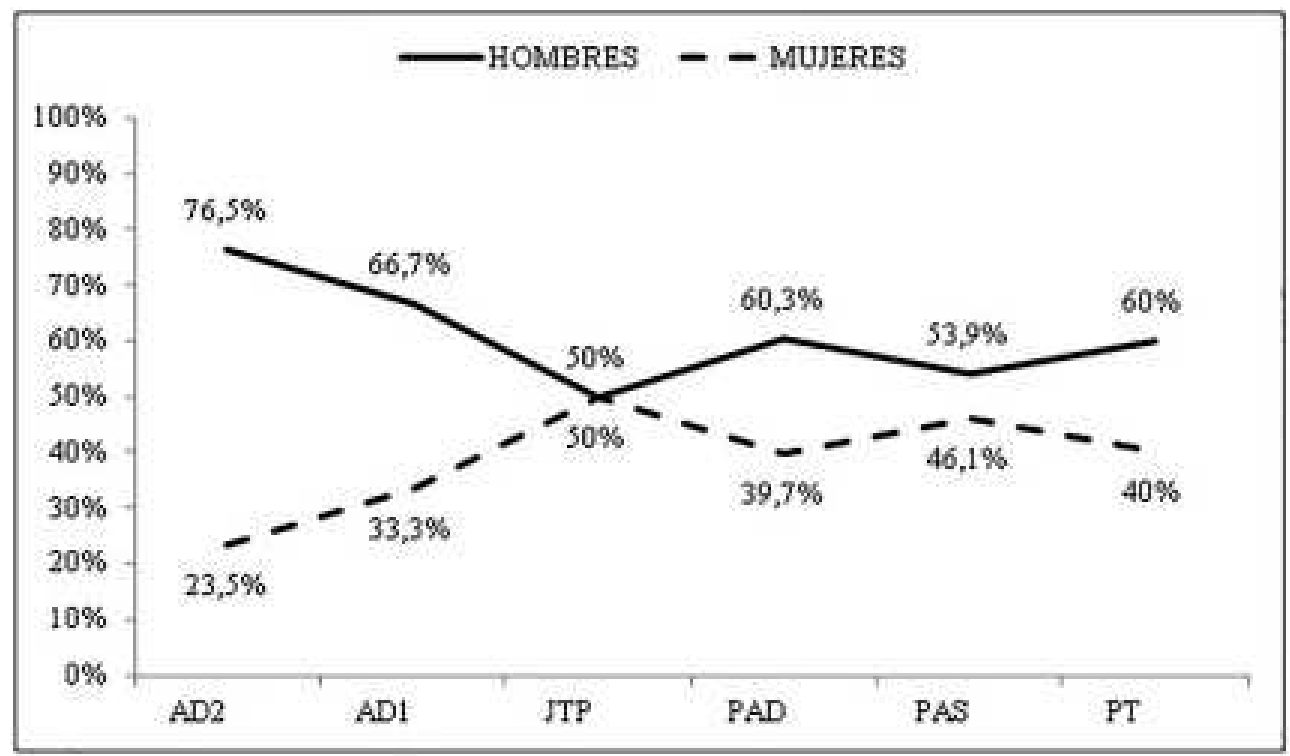

Fuente: Elaboración propia sobre datos de la UNSa (2015).

Se pasa de una representación del $62,5 \%$ de mujeres que defendieron la tesis doctoral en el ámbito de las Ciencias Aplicadas en la UNSa (SPU, 2014) a únicamente un $23,5 \%$ que accedieron a la categoría inferior de la escala docente, los/as auxiliares de segunda, en el año 2015. Paulatinamente, la participación femenina va ascendiendo hasta producirse una situación de igualdad porcentual entre los/as jefes/as de trabajos prácticos. No obstante, es a partir de ese momento, el que podemos definir como la puerta de entrada al cuerpo del profesorado docente, cuando las mujeres comienzan de nuevo a disminuir, constituyendo el $46,1 \%$ del profesorado asociado. Finalmente, en el cargo superior, el de profesorado titular, se produce una situación que podemos considerar de paridad, con un $40 \%$ de mujeres y un $60 \%$ de hombres (UNSa, 2015).

En conclusión, la Facultad de Ingeniería y los estudios en Ciencias Aplicadas de la UNSa se caracterizan por una menor participación femenina en el acceso tanto a los 
niveles iniciales de estudio (pregrado y grado) como en la carrera docente (auxiliares docentes). No obstante, los porcentajes de las mujeres aumentan a medida que se va avanzando en los estudios de posgrado y en las categorías de profesorado superior. A pesar de ello, se produce una diferencia considerable entre la cantidad de mujeres que finalizan la tesis doctoral en este ámbito y aquellas que consiguen superar las barreras y obstáculos para acceder a la carrera académica e investigadora en la Facultad de Ingeniería.

\section{Resultados}

A continuación se presenta el análisis de los resultados obtenidos tras la aplicación de la encuesta a la plantilla docente femenina de la Facultad de Ingeniería de la UNSa. Para ello, se han diferenciado cuatro subapartados, desarrollándose en cada uno de estos las principales conclusiones alcanzadas.

\subsection{Negación de discriminación directa o indirecta en la Universidad}

En primer lugar, de manera general, podemos afirmar que se produce una invisibilización de las barreras y obstáculos en el sistema universitario, sobre todo por parte de las docentes más jóvenes.

De este modo, cuando se consulta la opinión acerca de la existencia de discriminación directa o indirecta tanto en el momento del acceso a la carrera docente e investigadora como en la promoción hacia las categorías profesionales más altas en la UNSa, un $63,6 \%$ y un $68,2 \%$, respectivamente, consideraron que tales discriminaciones no existían (Gráfica 3).

Gráfica 3: Negación de discriminación directa o indirecta hacia la mujer en el acceso y promoción en la carrera docente e investigadora en la UNSa.

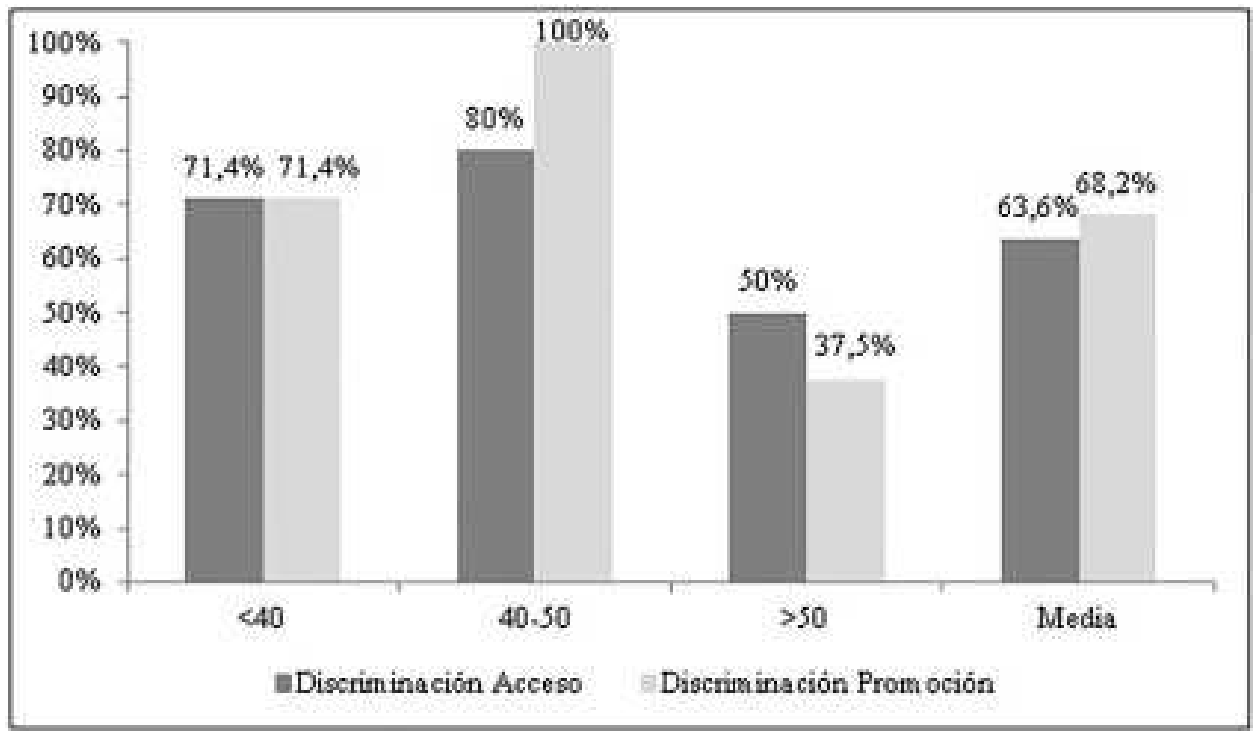

Fuente: Elaboración propia. 
Si analizamos los diferentes rangos de edad, llama la atención que únicamente en el caso de las mujeres mayores de 50 años se produce un equilibrio entre aquellas que confirmaron su existencia en el acceso y de las que lo negaron ( $50 \%$ en cada caso), mientras que el porcentaje asciende hasta el $71,4 \%$ en las menores de 40 años y el $80 \%$ de las mujeres de mediana edad.

Atendiendo a la discriminación de género en la promoción, las diferencias de opinión según la edad son significativas. Para el caso de las mujeres menores de 40 años los porcentajes replican a los anteriores: el $71,4 \%$ opinaron que no existía discriminación. El total de docentes de entre 40 y 50 años negó que se produjera tal situación. No obstante, el porcentaje de las mayores de 50 años que eligieron la respuesta 'no', descendió hasta el $37,5 \%$, por lo que se concluye que la gran mayoría es consciente de la problemática.

Como se observa, la diferencia de opinión más notable sobre la discriminación en el momento del acceso a la carrera y el proceso de promoción lo encontramos en las respuestas de las mujeres mayores de 50 años cuyo porcentaje disminuye en 12,5 puntos ( $50 \%$ negación de existencia de barreras en el acceso y $37,5 \%$ en la promoción). Por su parte, las mujeres de mediana edad, a pesar de que se deduce que son aquellas que se encuentran en el momento de promocionar y ascender en la carrera, son quienes a su vez muestran una mayor invisibilización y escasa concienciación ante la existencia de barreras y obstáculos en el proceso de promoción. Las más jóvenes, en su mayoría, consideran en ambos casos que tales discriminaciones no existen.

\subsection{Presencia femenina en los puestos de responsabilidad}

Al consultar la opinión acerca de la presencia femenina en los altos cargos de poder en la UNSa, prácticamente la mitad de estas opinaron que es bastante $(45,5 \%)$, y un $4,5 \%$ creyeron que es alta. Un $36,4 \%$ confirmaron ser acorde con el total de la plantilla femenina del profesorado y únicamente, un 13,6\% afirmaron que ésta es escasa.

Las docentes más satisfechas con la cantidad de mujeres en puestos de responsabilidad fueron aquellas de entre 40 y 50 años. De este modo, un $80 \%$ de ellas consideraron que son bastantes las mujeres en altos cargos. Sin embargo, este porcentaje cae hasta el $28,6 \%$ de las respuestas de las menores de 40 años y el $50 \%$ de las mayores de 50 años.

Las más jóvenes en su mayoría, un 57,1\%, opinaron que la representación de mujeres es acorde con el total de estas en la plantilla. Cabría esperar que fueran aquellas más críticas con la situación, puesto que estos altos cargos y puestos en aquellos escasos casos en los que están ocupados por mujeres, se trata habitualmente de docentes de mayor edad y con una trayectoria profesional más amplia. Así, podrían sentirse más identificadas y descontentas con el techo de cristal. Sin embargo, un escaso $14,3 \%$ confirmaron que la presencia es escasa.

Podemos afirmar que las más críticas son aquellas mayores de 50 años, quienes en un $25 \%$ seleccionaron la respuesta 'escasa' para definir la participación de las mujeres en los ámbitos de decisión y poder universitario.

\subsection{Percepción personal sobre la superación de barreras y obstáculos}

Una de las cuestiones planteadas más interesantes por la contradicción de los resultados en comparación con preguntas anteriores es aquella en la que se consultó si personalmente se habían encontrado con barreras y dificultades para promocionar a lo largo de su carrera académica. 
La diferencia entre las que respondieron afirmativamente y las que negaron haber experimentado esa situación es minúscula. A pesar de ello, fueron mayoría las que afirmaron haberse encontrado problemas para promocionar por el hecho de ser mujer. Se constata de esta forma la existencia del techo de cristal, negado hasta ahora en anteriores preguntas. De este modo, el $59,1 \%$ de las mujeres respondieron que sí, frente al $40,9 \%$ del no. Recordemos que más del $60 \%$ de las encuestadas negaron la existencia de procesos de discriminación directa o indirecta en la UNSa.

De nuevo se pueden observar diferencias más que notables por razón de edad, continuando con una menor concienciación y visibilización del problema por parte de las docentes más jóvenes. El mayor porcentaje de mujeres que refieren haber tenido dificultades y obstáculos a la hora de promocionar corresponde a las mayores de 50 años, en un $75 \%$ de los casos. Este porcentaje cae en 10 puntos hasta el $60 \%$ de profesoras entre 40 y 50 años. Finalmente, menos de la mitad de las menores de 40 años, el $42,9 \%$, respondieron afirmativamente (Gráfica 4).

Gráfica 4: Percepción sobre si se ha encontrado con barreras y dificultades para promocionar a lo largo de su carrera académica.

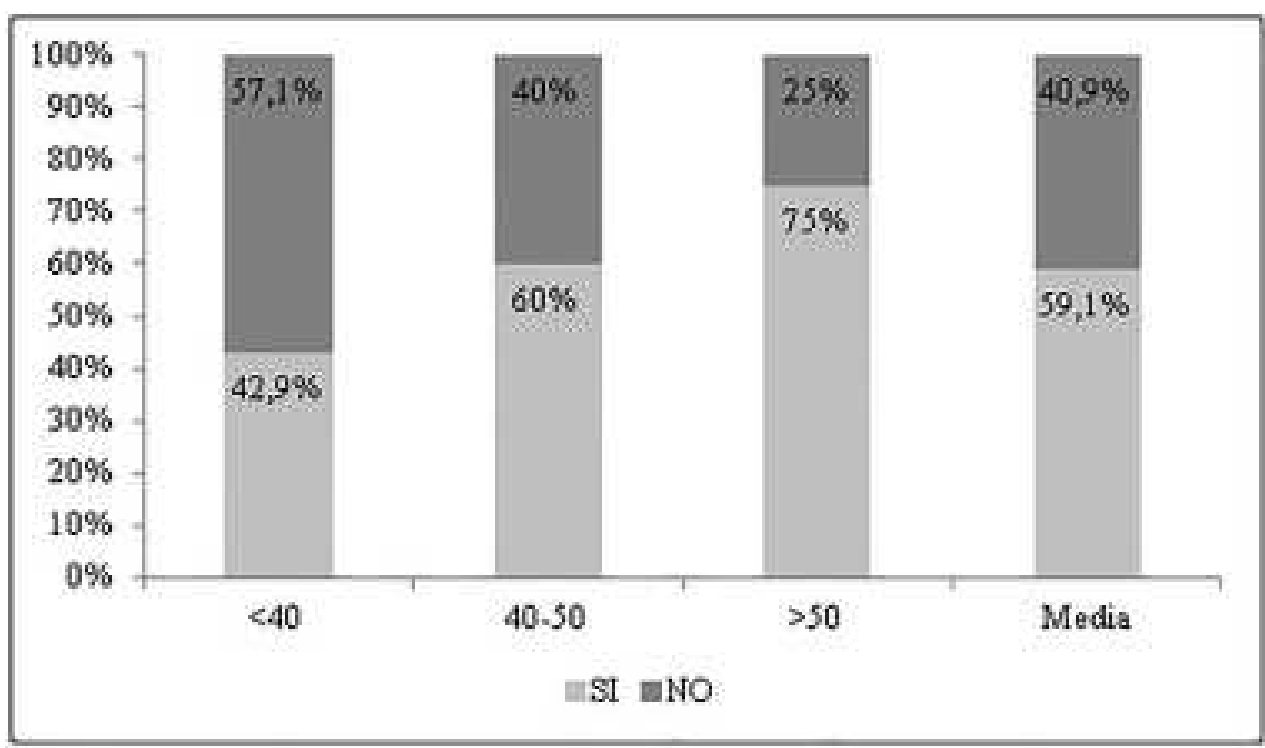

Fuente: Elaboración propia.

A pesar de que la mayor parte de las docentes de la Facultad de Ingeniería confirmaron haberse encontrado obstáculos durante su carrera académica, no existe un consenso cuando se les consulta si consideran que las barreras afectan de igual manera a sus compañeros varones. La diferencia porcentual entre aquellas que piensan que sí y las que opinan que no es mínima $(40,9 \%$ y $45,5 \%$, respectivamente). El porcentaje de mujeres que optaron por la tercera posibilidad de respuesta 'no sé/no contesto' es bastante elevada en comparación al resto de preguntas, con un 13,6\%.

De nuevo se produce una moderación en la opinión de las mujeres de edad media (40-50 años), siendo estas las que en mayor medida consideraron que las barreras y 
obstáculos afectan por igual a hombres y mujeres (60\%). Las mayores de 50 años continúan siendo las más críticas, o quizás, más conscientes de la problemática. Un $62,5 \%$ de estas opinaron que son ellas, las docentes, las que deben hacer frente en mayor ocasiones a dificultades. Las menores de 40 años, por su parte, fueron las que respondieron afirmativamente en un menor porcentaje $(28,6 \%)$, pero las que se mostraron en la misma medida más dubitativas a la hora de responder $(28,6 \%)$. Un $42,8 \%$ de estas opinaron que las mujeres debían de superar más barreras y obstáculos que los varones.

\subsection{Valoración de barreras y obstáculos del Techo de Cristal}

Finalmente, se presentó un listado con quince causas que dificultan el acceso de las mujeres a la categoría más alta, la de profesora titular. De manera general, podemos afirmar que las barreras personales, como la doble jornada laboral y el trabajo doméstico y de cuidados ( $\mathrm{P} 2$ y $\mathrm{P} 3$ ), sumadas a las organizacionales, como es la falta de apoyo y financiación ( $\mathrm{P} 13$ ) y de redes informales de poder (P9), y la persistencia de roles y estereotipos de género (P15), como barreras del contexto social, son las que, según las encuestadas, les afectan en mayor medida en su proceso de promoción (Tabla 1).

Tabla 1: Valoración de las barreras de promoción en una escala de 1 a 4.

\begin{tabular}{|c|c|c|c|c|c|c|c|c|c|c|c|c|c|c|c}
\cline { 2 - 14 } \multicolumn{1}{c|}{} & P1 & P2 & P3 & P4 & P5 & P6 & P7 & P8 & P9 & P10 & P11 & P12 & P13 & P14 & P15 \\
\hline$<40$ años & 1,4 & 2,9 & 2,9 & 2 & 2,1 & 2,4 & 2 & 1,6 & 2 & 1,4 & 1,7 & 1,6 & 2,9 & 2 & 2 \\
\hline 40 a 50 años & 2 & 2,6 & 2,6 & 1,8 & 1,8 & 1,6 & 1,2 & 1,8 & 2,8 & 2 & 1,6 & 1 & 1,5 & 1,5 & 1,8 \\
\hline$>50$ años & 1,6 & 1,8 & 2 & 1,7 & 1,6 & 2 & 1,6 & 2,4 & 2,3 & 2,1 & 2,5 & 2 & 2,6 & 2,7 & 2,9 \\
\hline $\begin{array}{c}\text { Promedio } \\
\text { total }\end{array}$ & 1,7 & 2,4 & 2,5 & 1,9 & 1,9 & 2,1 & 1,6 & 2 & 2,3 & 1,9 & 2 & 1,6 & 2,5 & 2,2 & 2,3 \\
\hline
\end{tabular}

Fuente: Elaboración propia.

Si atendemos a la variable edad, en primer lugar, las tres barreras con mayor puntuación por parte de las docentes más jóvenes coincidieron con las tres con mayor media, con una puntuación de 2,9 cada una. Estas fueron: la problemática de la ausencia de financiación y apoyo, el trabajo doméstico y de cuidados y la doble jornada laboral. A la primera de estas, los problemas y barreras derivados de la falta de apoyo y financiación (P13), le fueron otorgados por parte de las de mediana edad, únicamente, 1,8 puntos, mientras que las siguientes en edad la puntuaron con 2,6.

La doble jornada laboral (P3) tampoco congregó demasiado acuerdo como barrera: 2,9 puntos entre las jóvenes, 2,6 entre las de 40 a 50 años y 2 entre las mayores de 50. Consecuentemente, las cifras se mantienen cuando se consideran los/as hijos/as y las personas dependientes (P2) para las mujeres jóvenes y de mediana edad $(2,9$ y 2,6$)$. Sin embargo, los valores de las de más de 50 años disminuyeron hasta 1,8 puntos. En ambos casos se hace patente la mayor dificultad por parte de las mujeres más jóvenes para conciliar trabajo doméstico y de cuidados con el empleo en la universidad, principalmente porque se trata de aquellas donde la tasa de maternidad es más elevada así como las cargas derivadas de ésta.

A pesar de ello, llama la atención que al concretar el enunciado, acotando la causa del techo de cristal a las consecuencias derivadas de la escasa corresponsabilidad por parte de las parejas como son los problemas de conciliación (P1), el grado de acuerdo de las profesoras más jóvenes cae hasta 1,4 puntos. Se trata de casi 1,5 puntos menos que 
si lo comparamos con las puntuaciones otorgadas a la doble jornada laboral y el trabajo doméstico y de cuidados, pero sin mencionar la inexistente o baja corresponsabilidad por parte de la pareja. Por parte de las profesoras de más edad, la puntuación también disminuyó en esta cuestión, aunque de menor forma ( 2 para las de $40-50$ años y 1,6 para las mayores de 50 ).

En segundo lugar, por parte de las mujeres de entre 40 y 50 años de edad, el obstáculo más puntuado, y, por tanto, que presentó un mayor grado de acuerdo por parte de estas, fue aquel que afirmaba la existencia de redes informales de poder (P9) dentro de la academia como barrera de promoción para las mujeres, con 2,8 puntos ( 2 puntos por parte de las menores de 40 y 2,3 de las mayores de 50 ). De nuevo observamos como al denominar el mismo fenómeno de un modo más explícito, como sistemas de cooptación (P10), las valoraciones en el grado de acuerdo por parte del profesorado femenino -en general-, y del más joven -en particular-, disminuyen considerablemente. La diferencia en las mayores de 50 años es casi insignificante, puntuaron con 2,1 ; mientras que por su parte, las menores de 40 adjudicaron 0,6 puntos menos $(1,4)$ y las de mediana edad $0,8(2$ puntos).

En tercer lugar, las mujeres mayores de 50 años opinaron que los mayores obstáculos para promocionar lo constituyen aquellas barreras sociales como la existencia de estereotipos y roles de género (P15) -2,9- y la sociedad patriarcal (P14) -2,7-, alcanzando casi los 3 puntos de los máximos 4 posibles. En el caso de las más jóvenes, puntuaron este tipo de barreras con un 2, y las de mediana edad con 1,8. Esta diferencia generacional puede dar lugar a una mayor valoración por parte las profesoras de más edad o con más antigüedad, quiénes han vivido y se han educado en un contexto patriarcal inserto en una sociedad aún más machista (si cabe), aunque esto no quiere decir que se haya evolucionado en demasía en pro de la igualdad ni los estereotipos y roles sexistas de antaño hayan sido eliminados con el paso del tiempo.

Llama la atención que la discriminación directa (P8) por parte del profesorado o de la propia universidad como barrera para promocionar hasta las categorías más altas alcanzó una puntuación de 2 de media, a pesar de la negación de ésta de manera mayoritaria en anteriores cuestiones. De nuevo se constata la mayor visibilización y concienciación del problema por parte de las profesoras de más edad, siendo valorada con 2,4 , seguidas por las mujeres de entre 40 y 50 años por 1,8 y, las menores de 40 con 1,6 (de las menores puntuaciones, superado, únicamente, por los problemas de conciliación y la existencia de sistemas de cooptación, con una puntuación de 1,4).

Por último, con respecto a las barreras personales de índole interna, se confirma que son las profesoras más jóvenes aquellas que a las que en mayor medida su existencia le suponen un obstáculo para su promoción. En todas ellas (de la P4 a P7) les asignaron una puntuación de 2 o más de 2. Es en este tipo de barreras del techo de cristal donde se da un menor grado de acuerdo por parte de las mujeres mayores de 50 años, otorgándoles las valoraciones más bajas del total de causas presentadas (con excepción de la autorrestricción valorada con un 2). Las profesoras de entre 40 y 50 años fueron quiénes mostraron mayor disconformidad hacia estas causas internas del techo de cristal. De esto se desprende que son aquellas mujeres de mayor edad y con más experiencia las que presentan niveles de autoestima y seguridad y confianza en sí mismas más altos.

\section{Conclusiones}

Las mujeres se han constituido como una mayoría en los sistemas de Ciencia e Investigación así como en la educación superior argentina. Sin embargo, las cifras 
estadísticas muestran como estas continúan constituyendo una minoría entre los altos cargos de poder y responsabilidad, agrupándose principalmente en los puestos bases. De este modo, el presente trabajo se enmarca en la literatura existente como un esfuerzo tanto cualitativo como cuantitativo por contrastar la existencia de discriminación vertical y horizontal en la universidad (estudio descriptivo), por un lado; como las diferencias de percepción sobre la existencia de techos de cristal y sus causas (estudio explicativo), por el otro. Para ello se ha llevado a cabo un análisis estadístico de la plantilla de la UNSa, junto con una encuesta cuyas respuestas han sido contrastadas estadísticamente.

Los resultados muestran distintas apreciaciones del cuerpo docente femenino sobre esta problemática. En primer lugar, se confirma la invisibilización y negación de la existencia de procesos de discriminación tanto en el acceso como en la promoción desde la perspectiva de las propias mujeres involucradas -en mayor medida durante el acceso-. Los discursos públicos, e incluso oficiales por parte de las propias instituciones científicas, a menudo se centran en ensalzar la alta participación femenina en la Ciencia, sin embargo, se hace más ardua la tarea de encontrar experiencias y vivencias en las que las docentes e investigadoras confirmen haber tenido que enfrentarse a situaciones de discriminación directas e indirectas a lo largo de sus carreras.

El interés reside precisamente en que son las docentes más jóvenes quienes muestran una mayor negación hacia esta realidad, siendo sus opiniones estadísticamente significativas en comparación a sus compañeras con una mayor edad y antigüedad laboral. Parecen mostrar una escasa sensibilidad hacia su propia situación o quizás las más mayores, quienes poseen una trayectoria laboral más amplia y ocupan puestos más altos, han ido visibilizando los procesos de discriminación a medida que han tenido que ir superándolos a lo largo de sus historias de vida.

Sin embargo, como se observa en las estadísticas, el acceso de estas a la carrera académica se presenta difícil, pasándose de un $62,5 \%$ de mujeres que finalizan la tesis doctoral en el ámbito de las Ciencias Aplicadas (SPU, 2014) a únicamente un 25,5\% que ocupaban los cargos de Auxiliares de Segunda en la Facultad de Ingeniería de la UNSa (UNSa, 2015). A pesar de ello, más de la mitad de las docentes menores de 40 años consultadas negaban que las mujeres tuvieran que superar más barreras y obstáculos para promocionar que los hombres.

En segundo lugar, a pesar de que se observa un cierto pudor o rechazo a admitir la existencia de discriminación cuando se utiliza un estilo menos directo en la pregunta y unos términos más asépticos, por ejemplo, se emplean las palabras 'barreras y obstáculos para promocionar' en el lugar de 'discriminación vertical en la promoción' o 'techo de cristal', el porcentaje de mujeres que responden afirmativamente y confirman haber tenido que superar dichos obstáculos se eleva considerablemente. A pesar de ello, continúan considerando que la participación de la mujer en los altos cargos es bastante alta (aunque únicamente seis docentes del total de la plantilla de la Facultad de Ingeniería ocupen el cargo de profesora titular). Se trata, como definiera Gloria Bonder (1989) del fenómeno del 'espejismo de la igualdad', basado en una supuesta igualdad entre hombres y mujeres como consecuencia de una mayor participación femenina. Sin embargo, como hemos constatado a lo largo de este trabajo, ésta no se traduce ni en una representación igualitaria en los ámbitos de mayor poder, prestigio y responsabilidad, ni en una situación de igualdad con respecto a los obstáculos a superar durante la carrera profesional por parte de las mujeres.

En tercer lugar, acerca de las barreras que las mujeres conciben como causantes de este fenómeno, en general, son escasamente consideradas como tal por las docentes, principalmente por las más jóvenes. Se aprecian diferencias notables en el tipo de obstáculos que afectan en mayor medida a unas u otras según los diferentes rangos de edad.

14 Revista Estudos Feministas, Florianópolis, 26(2): e51339 
Podemos concluir que las mujeres más jóvenes y de mediana edad encuentran más dificultades que superar en el ámbito doméstico y familiar. Coinciden en sus vidas, simultáneamente, un periodo de asunción de mayores cargas derivadas de la maternidad y el cuidado de personas dependientes, junto con el momento de promocionar y seguir ascendiendo en su carrera académica e investigadora, por lo que la doble jornada laboral se presenta como un verdadero reto. Sin embargo, llama notablemente la atención la escasa puntuación asignada a los problemas derivados de la conciliación cuando se especifica y se incluye como causante la escasa o inexistente corresponsabilidad por parte de la pareja (en el caso de que existiese).

Además, la falta de financiación y apoyo y la existencia de redes informales de poder son otras de las causas valoradas en mayor medida por las docentes menores de 40 años y entre 40 y 50 años. Siguen perviviendo en la Universidad círculos de poder liderados por hombres cuya distancia o cercanía a éstos interfiere en la valoración de los/as posibles candidatos/as durante los procesos de promoción.

No obstante, las de mayor edad, quienes probablemente se encuentren en el culmen de su carrera profesional, se decantan por señalar los obstáculos y barreras de índole social, como la existencia de arraigados roles y estereotipos de género. Son estas quienes se han socializado y educado en una sociedad caracterizada por el machismo y el patriarcado, en mayor medida que en los tiempos actuales.

En resumen, parece ser que queda bastante por avanzar en la toma de conciencia de este fenómeno. No existe una correspondencia entre los datos que muestran las estadísticas y las percepciones personales. Por lo tanto, parece necesario retomar esta problemática y ponerla entre las principales preocupaciones de la agenda política. Además, se debe continuar avanzando en el diseño e implementación de medidas de intervención en pro de la igualdad de género.

\section{Referencias}

ALONSO, María José. "Las académicas. Profesorado universitario y género". Revista de Educación, Madrid, n. 328, p. 465-475. 2002.

ANTÓN, Susana. Informe: Académicas en la Universidad de Alicante. Cuadernos de Trabajo de Investigación 12. Alicante: Universidad de Alicante, 2005.

BAILYN, Lotte. "Academic careers and gender equity: Lessons learned from MIT". Gender, Work and Organization, Cambridge, v. 10, n. 2, p. 137-153. 2003.

BARBERÁ, Esther; RAMOS, Amparo; SARRIÓ, Maite y CANDELA, Carlos. "Más allá del 'techo de cristal'. Diversidad de género". Revista del Ministerio de Trabajo y Asuntos Sociales, Madrid, n. 40, p. 55-68. 2002.

BAXTER, Janeen y WRIGHT, Erik Olin. "The glass ceiling hypothesis: A comparative study of the United States, Sweden, and Australia". Gender and Society, Madison, v. 14, n. 2, p. 275294. 2000.

BONDER, Gloria. "Las mujeres y la educación en la Argentina: realidades, ficciones y conflictos de las mujeres universitarias". In: GIBERTI, Eva y FERNÁNDEZ, Ana María (comp.). La mujer y la violencia invisible. Buenos Aires: Sudamericana. 1989. p. 37-61.

BONDER, Gloria (Dir.); BENEDETTI, Anabella; FLORES, Patricia y MAGARIÑOS, Paula. Mujeres que hacen ciencia y tecnología en Argentina: una década de avances y retos pendientes. 2003-2013. Cátedra Regional UNESCO Mujer, Ciencia y Tecnología en América Latina (FLACSO, Argentina). 2013.

COMISIÓN EUROPEA. She Figures 2015. Gender in research and innovation. Bruselas: Dirección General de Investigación e Innovación. 2016. 
DE PABLO, Flora. "Científicas y Tecnólogas: Especies a proteger". In: DE PABLO, Flora (Ed.). Desequilibrios de género en ciencia y tecnología. Sevilla: Editorial ArCiBel, 2006. p. 115-121.

ESCOLANO, Esther. "Discriminación en un medio meritocrático: las profesoras en la universidad española". Revista Mexicana de Sociología, México, v. 68, n. 2, p. 231-263. 2006.

ESTÉBANEZ, María Elina. "Género y profesión en el análisis de la ciencia argentina". In: GERVASONI, Juana. (Comp.). Foro nacional interdisciplinario mujeres en ciencia, tecnología y sociedad, Bariloche: CNEA. 2010.

ESTÉBANEZ, María Elina (Coord.), DE FILIPPO, Daniela y SERIAL, Alejandra. La participación de la mujer en el sistema de Ciencia y Tecnología en Argentina. Buenos Aires: UNESCO-OEI. 2003.

FERNÁNDEZ, Concepción. "La mujer en la Universidad española. Docencia, investigación y poder. Datos y aspectos cualitativos". Revista de Educación, Madrid, n. 290, p. 161-171. 1989.

FLECHA, Consuelo y PALERMO, Alicia I (Coord.). Mujeres y universidad en España y América Latina. Buenos Aires: Miño y Dávila Editores. 2008.

GARCÍA, Susana V. "Ni solas ni resignadas: la participación femenina en las actividades científico-académicas de la Argentina en los inicios del siglo XX". Cadernos Pagu, Campinas, n. 27, p. 133-172. 2006.

GARCÍA DE LEÓN, Ma Antonia. "Las profesoras universitarias: el caso de una élite discriminada". Revista Complutense de Educación, Madrid, v. 1, n. 3, p. 355-372. 1990.

GARCÍA DE LEÓN, Ma Antonia y GARCÍA DE CORTÁZAR, Ma Luisa (Comps.). Las académicas. Profesorado universitario y género. Madrid: Instituto de la Mujer. 2001.

GUIL, Ana. Techos de cristal en la Universidad de Sevilla. Madrid: Ministerio de Trabajo y Asuntos Sociales, Instituto de la Mujer. 2005.

GUIL, Ana. "Mujeres y ciencia: techos de cristal". Eccos Revista Científica, São Paulo, v. 10, n. 1, p. 213-232. 2008.

GUIL, Ana, SOLANO, Ana y ÁLVAREZ, Manuela. La situación de las mujeres en las universidades públicas andaluzas. Sevilla: Consejo Económico y Social de la Junta de Andalucía. 2005.

ION, Georgeta; DURÁN, Ma del Mar y BERNABEU, Ma Dolores. "El profesorado y su percepción sobre la igualdad de género en la universidad". Revista Complutense de Educacion, Madrid, v. 24, n. 1, p. 123-140. 2013.

JACKSON, Jerlando F.L. y O'CALLAGHAN, Elizabeth M. "What do we know about glass ceiling effects? A taxonomy and critical review to inform higher education research". Research in Higher Education, Talahassee, v. 50, n. 5, p. 460-482. 2009.

LLORENT, Vicente y COBANO, Verónica. "La mujer en los órganos de gobierno de la Universidad de Sevilla". Revista Estudos Feministas, Florianópolis, v. 25, n. 1, p. 241-262. 2017.

MAGLIE, Graciela y FRINCHABOY, Mónica. Situación educativa de las mujeres en Argentina. Buenos Aires: UNICEF, 1988.

MATUS, Mauricio y GALLEGO, Nazareth. "Techo de cristal. Si no lo veo no lo creo". Revista Complutense de Educación, Madrid, v. 26, n. 3, p. 611-626. 2015.

MORRISON, Ann M., WHITE, Randall y VAN VELSOR, Ellen. Breaking the Glass Ceiling: Can Women Reach the Top of America's Largest Corporations? New York: Addison-Wesley. 1987.

OGBOGU, Christiana O. "Gender factors affecting female labour input in the Nigerian University System". Gender and Behaviour, AJOL, v. 8, n. 1, p. 2666-2677. 2010.

PALERMO, Alicia I. "La participación de las mujeres en la universidad". La Aljaba, La Pampa, Luján y Comahue, v. 3, p. 94-110. 1998.

16 Revista Estudos Feministas, Florianópolis, 26(2): e51339 
PALERMO, Alicia I. "El acceso de las mujeres a la educación universitaria". Revista Argentina de Sociología, Buenos Aires, v. 4, n. 7, p. 11-46. 2006.

PASTOR, Inma; BELZUNEGUI, Ángel; MORENO, Blanca y MAÑAS, Carmen. "La igualtat d'oportunitats a la universitat: les percepcions del PDI". Papers: Revista de Sociología, Barcelona, v. 95, n. 2, p. 457-481. 2010.

PANAIA, Marta. "La inclusión de la mujer en la profesión de ingeniería". Revista Virajes, Manizales, v. 16, n. 1, p. 19-43. 2014.

PÉREZ Eulalia; SANTESMASES, Ma Jesús y ALCALÁ, Paloma (Comp.). Mujer y ciencia. La situación de las mujeres investigadoras en el sistema español de ciencia y tecnología. Madrid: Ministerio de Educación y Ciencia, Fundación Española para la Ciencia y Tecnología. 2005.

PROBERT, Belinda. "I just couldn't fit in: Gender and Unequal Outcomes in Academic Careers". Gender Work and Organization, New Jersey (Wiley Online Library), v. 12, n. 1, p. 50-72. 2005.

PUY, Ana. (Comp.). Barreras a la promoción de las mujeres a puestos de alto status en la universidad. Madrid: Ministerio de lgualdad. 2008.

REBOLLEDO, Loreto y ESPINOZA, Ma Paulina. "Género, Universidad e Investigación. Una tríada compleja". Revista Anales, Santiago de Chile, n. 11, p. 155-171. 2016.

ROJO, Sofía y TUMINI, Lucía. "Inequidades de género en el mercado de trabajo de la Argentina: las brechas salariales". Revista de Trabajo, Buenos Aires, v. 4, n. 6, p. 53-70. 2008.

SALIDO, Olga. "Las oportunidades de las mujeres en una estructura social cambiante. Documento de Trabajo". Documentos de trabajo. CSIC. Unidad de Políticas Comparadas, v. 2. 2002.

SÁNCHEZ, Inés, DE LA RICA, Sara y DOLADO, Juan José. Libro Blanco sobre la situación de las mujeres en la ciencia española. Madrid: Unidad de Mujeres y Ciencia. Ministerio de Ciencia e Innovación. 2011.

SISTEMA RHUN. Área de Recursos Humanos de las Universidades Nacionales. Argentina: Dirección Nacional de Presupuesto e Información Universitaria. 2013.

SPU. Sistema de consultas de estadísticas universitarias. Argentina: Ministerio de Educación de la Nación. 2014.

TOMÁS, Marina y GUILLAMÓN, Cristina. "Las barreras y los obstáculos en el acceso de las profesoras universitarias a los cargos de gestión académica". Revista de Educación, Madrid, n. 350, p. 253-275. 2009

TORRES, Obdulia y PAU, Bernadette. "Techo de cristal y suelo pegajoso: La situación de la mujer en los sistemas alemán y español de ciencia y tecnología". CTS: Revista Iberoamericana de Ciencia, Tecnología y Sociedad, Buenos Aires, v. 6, n. 18, p. 35-59. 2012.

UNSa. Estadísticas de la planta docente de la Universidad Nacional de Salta. Ciudad de Salta: Departamento de Estadísticas Universitarias. 2015.

[Recebido em 26/06/2017

e aprovado em 23/1 1/2017]

Gender Discrimination in the Argentine University System

Abstract: Women are the majority both among the students and in the teaching staff at the Argentine universities. However, they appear grouped mainly in certain areas and barely access the higher upper categories. There is a horizontal and a vertical pattern of gender segregation. Our objective is to study the participation of women and to deepen the perception of gender 
discrimination. Firstly, a descriptive analysis of the faculty of the National University of Salta (UNSa) is carried out. Secondly, the results of a survey applied to the female staff of the Faculty of Engineering of the UNSa are analyzed. There is a low perception and awareness of the problem, especially on the part of the teachers of minor age and they point out certain barriers as the main obstacles in the academic career.

Keywords: Women; Occupational segregation; Science; Argentina

Nazareth Gallego Morón é diplomada en Educación Social y Trabajo Social. Máster en Género e lgualdad y doctoranda en el Programa Desarrollo y Ciudadanía con la tesis titulada "Techo de cristal en las Universidades Españolas". Actualmente disfruta de un contrato predoctoral de Formación del Profesorado Universitario (FPU) concedido por el Ministerio de Educación. Miembro del Observatorio GEP\&DO (Gender, Economics, Politics and Development Observatory) y del Grupo de Investigación EcoEcoFem (SEJ507) "Economía Ecológica, Feminista y Desarrollo" de la Universidad Pablo de Olavide. Sus principales líneas de investigación giran en torno al análisis de la discriminación de género y participación de las mujeres en los mercados de trabajo, la discriminación laboral por género en ámbito universitario, los usos del tiempo, y la participación de las mujeres en la educación superior. 